\title{
Extreme Thrombocytosis: A Unique Constellation of Reactive Thrombocytosis, Iron Deficiency Anemia and Atypical Celiac Disease
}

\author{
Hamza Hashmi MD*, Umayr Azimi MD \\ Department Of Internal Medicine, Grand Rapids Medical Education Partners, Grand Rapids, Michigan \\ *Corresponding author: hamza.hashmi@grmep.com
}

Received April 29, 2015; Revised May 17, 2015; Accepted May 31, 2015

\begin{abstract}
Reactive thrombocytosis refers to elevated platelet count in the absence of a myeloproliferative or myelodysplastic syndrome and is secondary to a medical or surgical condition. Extreme thrombocytosis $(>1000)$ is unlikely to be autonomous in origin and an underlying reactive process should be strongly suspected. A 22 year old apparently healthy male presented with fatigue of five months duration. Labs showed marked hypochromic microcytic anemia with hemoglobin of $5 \mathrm{~g} / \mathrm{dL}$, extremely high platelet count of $1600 \times 10^{9}$ and undetectable ferritin. Upper and lower GI scopes failed to reveal any source of bleeding. Ultrasound abdomen revealed mildly enlarged spleen. Besides markedly increased megakaryopoesis, erythropoesis and low iron stores; bone marrow biopsy did not reveal any myelodysplasia. Patient was transfused 4 units of Packed RBC with rapid improvement of hemoglobin and platelet count within one week. Villous atrophy on duodenal biopsy and elevated tissue transglutaminase antibody levels confirmed diagnosis of celiac disease. Patient was started on iron supplements and gluten free diet. Follow up in 3 months revealed completely normal hemoglobin, ferritin level and platelet count. Extreme thrombocytosis should raise suspicion for a secondary cause rather than a primary myeloproliferative disorder. Iron deficiency anemia, a relatively rare but benign cause should be strongly considered in work up of reactive thrombocytosis. Atypical forms of celiac disease can be relatively asymptomatic. If clinical suspicion is high serum antibody assay and intestinal biopsy should be considered. The case illustrates rare constellation of extreme thrombocytosis from iron deficiency anemia in an otherwise asymptomatic celiac disease patient.
\end{abstract}

Keywords: extreme thrombocytosis, reactive thrombocytosis, iron deficiency anemia, celiac disease

Cite This Article: Hamza Hashmi MD, and Umayr Azimi MD, "Extreme Thrombocytosis: A Unique Constellation of Reactive Thrombocytosis, Iron Deficiency Anemia and Atypical Celiac Disease." American Journal of Medical Case Reports, vol. 3, no. 6 (2015): 184-186. doi: 10.12691/ajmcr-3-6-10.

\section{Introduction}

Elevated platelet count can be autonomous or reactive in origin. Autonomous thrombocytosis refers to primary myeloproliferative or myelodysplastic process in the bone marrow. Reactive thrombocytosis refers to elevated platelet count in the absence of a myeloproliferative or myelodysplastic syndrome. It is secondary to a medical or surgical condition. Extreme thrombocytosis (> 1 million) is unlikely to be autonomous in origin and an underlying reactive process should be strongly suspected. Thrombocytosis secondary to a reactive process is easily reversible.

\section{Case Description}

A 22 year old male with no significant past medical or surgical history presented to the primary care physician with the chief complaint of gradually worsening fatigue of five months duration. Fatigue was associated with unintentional weight loss of about $15 \mathrm{~kg}$ in six months. There were no other constitutional symptoms. There was no history of any recent travels, tick bites or sick contacts. Patient was sexually active with one partner and denied any unprotected sexual encounters. He did not have any known medical problems and did not take any prescribed or over the counter medication or supplements.

Physical exam revealed slightly cachectic appearance with a body mass index of 21.5. Marked pallor of mucous membranes was seen. Rectal examination did not reveal any obvious source of gastrointestinal bleeding.

Given concerns for anemia he was sent to the emergency department. Laboratory studies showed marked hypochromic microcytic anemia with hemoglobin of $5 \mathrm{~g} / \mathrm{dl}, \mathrm{MCV}$ of $60 \mathrm{fl}$ and extremely high platelet count of $1600 \times 10^{9} / \mathrm{L}$. Patient was admitted to the hospital for further work up and treatment of anemia. Peripheral smear revealed anisocytosis and poikilocytosis but no evidence of any blasts or schistocytes. Iron studies were done and were found to markedly abnormal and consistent with iron deficiency anemia with an undetectableferritin level of less than $15 \mathrm{pg} / \mathrm{ml}$, low iron level of $4.0 \mu \mathrm{mol} / 1$ (normal 
range 8.0-26 $\mu \mathrm{mol} / \mathrm{l})$, elevated transferrin level of $5.0 \mathrm{~g} / \mathrm{l}$ (normal range 2.0-3.6 g/l) and low transferrin saturation of $2.0 \%$ (normal range 16.0-45\%). Serum erythropoietin levelswere found to be markedly elevated at $900 \mathrm{MU} / \mathrm{ml}$. Markers of inflammation and hemolysis including ESR, CRP, LDH and haptoglobin were normal. Vitamin B12, folate, calcium and Vitamin D levels were also found to be within normal range. Patient underwent esophagogastroduodenoscopy and colonoscopy both of which failed to reveal any obvious source of bleeding. Ultrasound abdomen revealed mildly enlarged spleen. Bone marrow biopsy showed markedly increased megakaryopoesis and erythropoiesis but no evidence of a dysplastic process and normal cytogenetic.

Patient was transfused four units of Packed Red blood cells with rapid improvement of his hemoglobin and hematocrit on day 2 of admission. His platelet count also normalized on day 4 of hospitalization. In absence of any identifiable cause of iron deficiency anemia and unexplained weight loss, patient underwent testing for celiac disease. His tissue transglutaminase antibody levels came back elevated at $12 \mathrm{U} / \mathrm{ml}$ and duodenal biopsy showed characteristic villous atrophy and lymphocytic infiltrates seen in celiac disease both of which confirmed diagnosis of celiac disease. Patient was discharged with iron supplements and a gluten free diet. His hemoglobin and platelet count upon discharge were $12 \mathrm{~g} / \mathrm{dl}$ and $390 \mathrm{x}$ $10 \%$ l. Follow up in 3 months revealed completely normal hemoglobin, ferritin leveland platelet count.

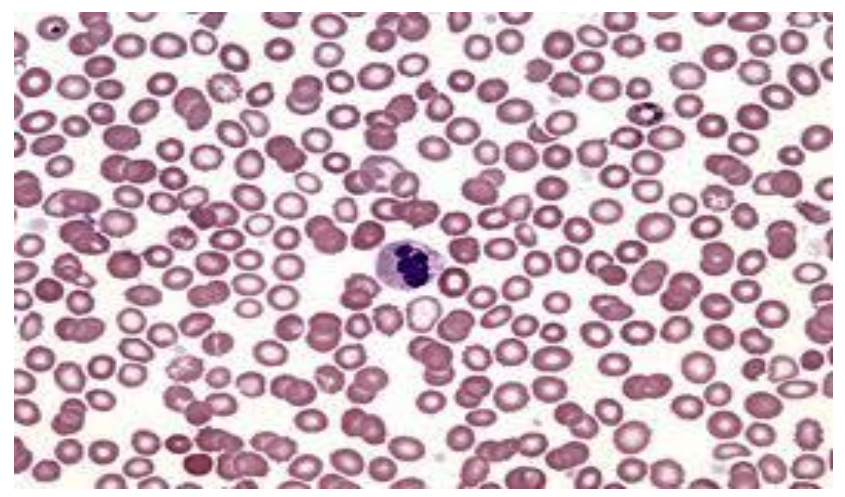

A. Peripheral smear showing hypochromic microcytic anemia

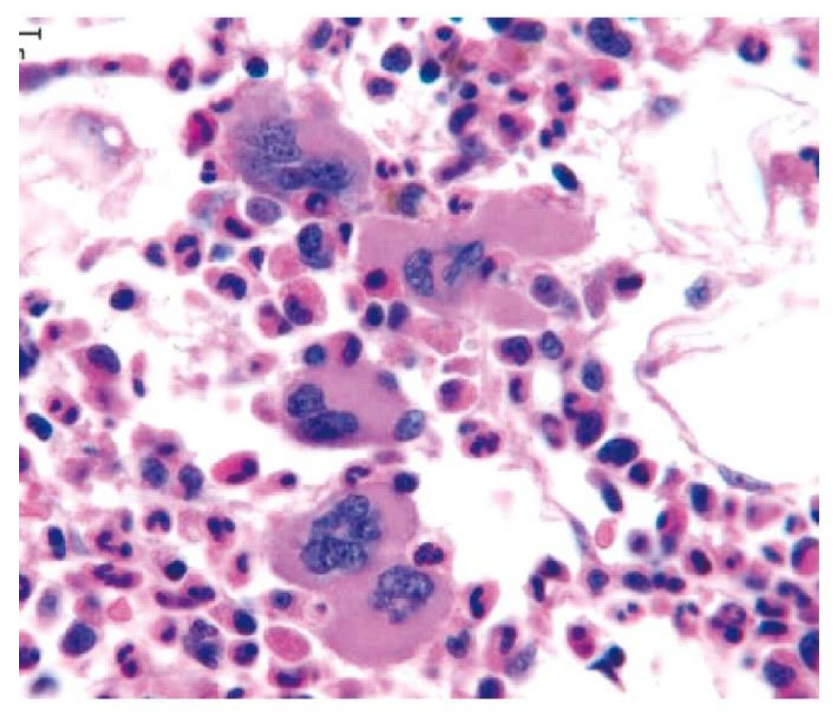

B. Bone marrow biopsy showing increased megakarpoises

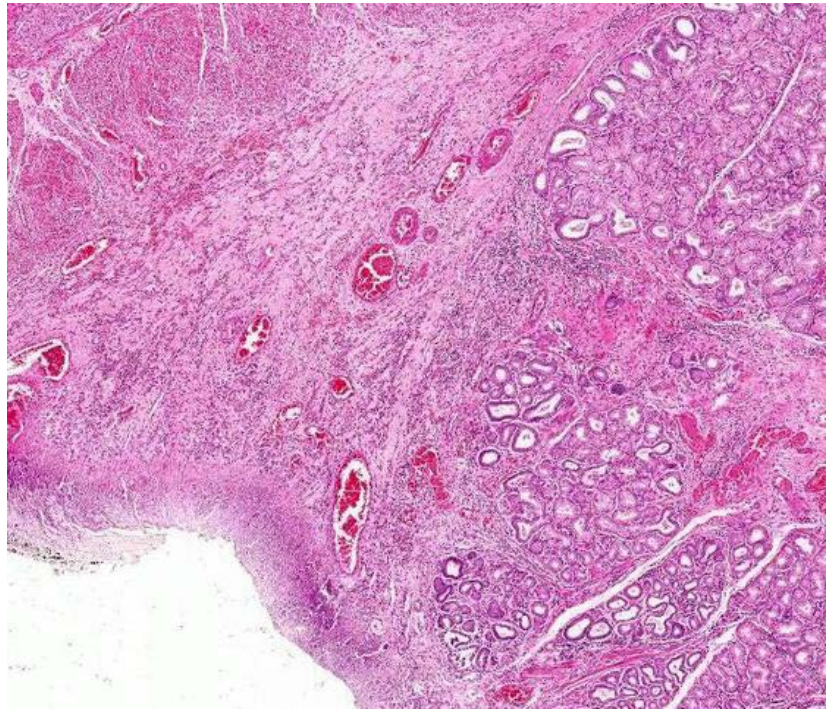

C. Duodenal Biopsy showing villous atrophy

\section{Discussion}

Extreme thrombocytosis is defined as platelet count greater than $1000 \times 10^{9}$. It can be reactive or autonomous in origin. It can present with vasomotor, bleeding or thrombotic complications [1,4]. Although these complication are common in patients with myeloproliferative or myelodysplastic syndromes; they are relatively rarely seen in reactive thrombocytosis. Autonomous causes primarily include myeloproliferative bone marrow disorder like Chronic Myeloid Leukemia, Polycythemia Vera or Essential Thrombocytosis. Certain Myelodysplastic Syndrome especially 5q-syndrome are also associated with thrombocytosis [2]. Reactive causes include occult malignancy, chronic infectious or inflammatory disease, secondary after hemorrhage or hemolytic crisis, asplenia and iron deficiency anemia [2].

In addition to ruling out myeloproliferative disorders with bone marrow biopsy; diagnostic work up of thrombocytosis should involve a detailed history, physical exam and laboratory studiesto identify any underlying reactive process [2]. Laboratory studies should include complete blood count, peripheral smear, iron studies, markers of inflammation and hemolysis and appropriate imaging studies guided by history and physical examination. Bone marrow is often a necessity to rule out any malignant hematological disorder.

Usually the thrombocytosis from iron deficiency anemia does not exceed $700 \times 10^{9}$, this care is unique as the platelet count exceeded well beyond extreme thrombocytosis range of 1 million [1]. The exact mechanism of thrombocytosis in iron deficiency anemia remains unclear. One hypothesis suggests that cross reaction between erythropoietin and thrombopoietin receptors due to structural similarity leads to increase stimulation of platelet production [5]. Another hypothesis lays emphasis on synergistic effect of erythropoietin and thrombopoietin on progenitor cells [6]. Both hypothesis fail to fully explain the phenomenon.

This case also demonstrates that presentation of celiac disease is variable ranging fromtypical forms presenting with classical manifestations of diarrhea, malabsorption and weight loss to atypical forms presenting with subtle 
symptoms or complete absence of symptoms [3]. If suspicion remains high, diagnostic testing withserum antibody assay and intestinal biopsy is warranted. Thrombocytosis in celiac disease could be secondary to inflammation, iron deficiency or functional asplenia.

\section{Conclusion}

The case illustrates rare constellation of extreme thrombocytosis from iron deficiency anemia in an otherwise asymptomatic celiac disease patient. Extreme thrombocytosis should raise suspicion about secondary cause rather than a primary myeloproliferative process. Although a rare cause, iron deficiency anemia should be considered in work up of reactive thrombocytosis as repleting iron stores corrects thrombocytosis. In the absence of any identifiable cause of iron deficiency anemia serum antibody testing and intestinal biopsy should be considered even when classical manifestations of celiac disease are missing.

\section{Acknowledgements}

None.

\section{Statement of Competing Interests}

The authors do not have any statement of competing interests.

\section{Abbreviations}

MCV : $\quad$ Mean corpuscular volume

LDH : $\quad$ Lactate dehydrogenase

CRP: $\quad C$ reactive protein.

\section{References}

[1] Buss DH, Cashell AW, O'Connor ML, Richards F: Occurrence, etiology and clinical significance of extreme thrombocytosis: a study of 280 cases. Am J Med 1994, 96:247-253.

[2] Sanchez S, Ewton A: Essential thrombocythemia: a review of diagnostic and pathologic features. Arch Pathol Lab Med 2006, 130(8):1144-1150.

[3] Halfdanarson TR, Litzow MR, Murray JA: Hematologic manifestations of celiac disease.Blood 2007, 109(2):412-421.

[4] Schafer AI: Thrombocytosis. N Engl J Med 2004, 350(12):12111219.

[5] Bilic E, Bilic E: Amino acid sequence homology of thrombopoietin and erythropoietin may explain thrombocytosis in children with iron deficiency anemia. J PediatrHematolOncol 2003, 25(8):675-676.

[6] Akan H, Guven N, Aydogdu I, Arat M, Beksac M, Dalva K: Thrombopoietic cytokines in patients with iron deficiency anemia with or without thrombocytosis. ActaHaematol 2000, 103(3):152156. 